\title{
Puzzling Dyspnea Caused by Respiratory Muscle Weakness
}

\author{
Adriana J. Pavletic and Oleb Hnatiuk
}

Dyspnea is common in advanced stages of neuromuscular disorders, but it is infrequently the presenting symptom. However, dyspnea is a frequent complaint in a primary care setting but is rarely caused by a respiratory muscle weakness. Consequently, the diagnosis of respiratory muscle weakness often is delayed. First symptoms may occur when respiratory muscles are under increased load, such as when standing in the water higher than the chest, swimming, or in the supine position. We describe a patient in whom dyspnea was the first symptom of amyotrophic lateral sclerosis to remind clinicians of clinical features of respiratory muscle weakness and to help avoid the delay in diagnosis. ( $\mathrm{J}$ Am Board Fam Med 2012;25:396-397.)

Keywords: ALS (Amyotrophic Lateral Sclerosis), Dyspnea, Neuromuscular Disorders, Respiratory Muscles

Although dyspnea is a frequent complaint in a primary care setting, it is rarely caused by a respiratory muscle weakness. Consequently, physicians often are not familiar with the clinical presentation of respiratory muscle dysfunction and it may be wrongly attributed to other causes. ${ }^{1}$ To remind physicians about this potentially puzzling condition, we describe a patient in whom dyspnea was the first symptom of amyotrophic lateral sclerosis (ALS).

A 79-year-old, white, nonsmoking, physically fit man complained of progressive shortness of breath for 1 year. He had well-controlled hypertension, type 2 diabetes mellitus, and dyslipidemia, for which he took lisinopril, metformin, and simvasta-

This article was externally peer reviewed.

Submitted 11 July 2011; revised 16 November 2011; accepted 23 November 2011.

From Office of the Clinical Director, National Institute of Mental Health, Bethesda, MD (AJP); and Walter Reed Army Medical Center, Washington, DC (OH).

Funding: This work was supported by the Intramural Research Program of the National Institutes of Health.

Conflict of interest: none declared.

Disclaimer: The views expressed in this article do not necessarily represent the views of the National Institute of Mental Health, National Institutes of Health, the United States Department of Health and Human Services, or the United States Government.

Corresponding author: Adriana J. Pavletic, National Institute of Mental Health, Office of the Clinical Director, 10 Center Drive, Bethesda, MD 20892 (E-mail: pavletia@mail.nih.gov). tin. He had no history of pulmonary disease, heart disease, pet exposure, or recent travel.

Initially, he was unable to take a deep breath when swimming or standing in a pool with water higher than his chest. However, he was able to walk 3 miles without difficulty. Later, his dyspnea was accompanied by exertional dyspnea, orthopnea, and an unexplained 20-lb weight loss. He had no cough, fever, night sweats, chest pain, or leg pain.

Initial physical examination, laboratory assessment, chest radiograph, and cardiopulmonary exercise testing were normal. Pulmonary function testing revealed the following: forced expiratory volume in 1 second $\left(\mathrm{FEV}_{1}\right), 1.88 \mathrm{~L}(68 \%)$; forced vital capacity (FVC), $2.42 \mathrm{~L}(66 \%)$; and $\mathrm{FEV}_{1}$ / FVC, $78 \%$. There was no change after bronchodilator use. His total lung capacity was $5.37 \mathrm{~L}$ (87\%), and the diffusing capacity of the lung for carbon monoxide was $19.4 \mathrm{~mL} / \mathrm{min} / \mathrm{mm} \mathrm{Hg}(106 \%)$.

One year after the onset of symptoms our patient experienced right leg weakness, throat irritation, and difficulty swallowing. Almost 1.5 years into his illness, the patient developed dysarthria, peripheral muscle weakness, muscle wasting, and fasciculations. A diagnosis of ALS was made.

Our patient's respiratory function was reassessed; his upright vital capacity was fairly preserved at $71 \%$ but fell to $36 \%$ in the supine position. His maximal inspiratory pressure was -43 (normal, -65 to -90 ) and his maximal expiratory 
pressure was +68 (normal, +140 to +190$).^{2}$ His daytime fatigue temporarily improved with the nighttime use of bilevel positive airway pressure, but his illness relentlessly progressed and he died of respiratory failure 3 years after the onset of symptoms.

Respiratory muscle dysfunction is common among patients with many neuromuscular disorders. ${ }^{3}$ It is rarely a presenting symptom and therefore often goes unrecognized. ${ }^{1}$ Clinical presentation can be chronic progressive (eg, ALS), acute (eg, Guillain-Barré syndrome), or chronic relapsing (eg, myasthenia gravis, multiple sclerosis). Clinical course, prognosis, and treatment are variable and depend on the underlying disease. ${ }^{3}$

Patients with motor neuron disease typically complain of the inability to take a deep breath, which may be confused with anxiety. ${ }^{1}$ However, their symptoms are worse in a supine position, which is not a feature of psychogenic dyspnea. ${ }^{1}$ Our patient's initial symptom of dyspnea while immersed in water likely was caused by diaphragmatic dysfunction. $^{4}$

In one study involving patients with multiple sclerosis, a clinical assessment index was found to better predict respiratory muscle weakness than spirometry. ${ }^{5}$ This index comprised of patients' reports of a weakened cough and difficulty clearing pulmonary secretions, weakened cough as rated by the examiner, and the counting test (value reached when the patient counts aloud on a single exhalation after maximum inspiratory effort; normal $\geq 30)^{5}$
Pulmonary function tests in patients with advanced respiratory muscle weakness have a restrictive pattern (reduced FVC and total lung capacity, normal $\mathrm{FEV}_{1} / \mathrm{FVC}$ ) and reduced maximal inspiratory pressure and maximal expiratory pressure. ${ }^{3}$ Vital capacity measured in the supine position is usually lower than in the upright position.

Respiratory muscle weakness should be considered in patients whose dyspnea cannot be explained by pulmonary disease, cardiac disease, anemia, or deconditioning. First symptoms may occur when respiratory muscles are under increased load, such as when standing in deep water, swimming, or in the supine position. ${ }^{4}$

\section{References}

1. Nightingale S, Bates D, Bateman DE, Hudgson P, Ellis DA, Gibson GJ. Enigmatic dyspnoea: an unusual presentation of motor neurone disease. Lancet 1982;1:933-5.

2. Enrighi PL, Kronmal RA, Manolino TA, Schenker MP, Hyatt RE. Respiratory muscle strength in the elderly. Correlates and reference values. Am J Resp Crit Care Med 1994;149: 430-8.

3. Perrin C, Unterborn JN, D'Ambrosio C, Hill NS. Pulmonary complications of chronic neuromuscular diseases and their management. Muscle Nerve 2004; 29:5-27.

4. Mier AK, Brophy C, Green M. Out of depth, out of breath. BMJ 1986;292:1495-6.

5. Smeltzer SC, Skumick JH, Troiano R, Cook SD, Duran $\mathrm{W}$, Lavietes $\mathrm{MH}$. Respiratory function in multiple sclerosis: utility of clinical assessment of respiratory muscle function. Chest 1992;101:479-84. 